\title{
Quinas en la Flora de la Real Expedición Botánica del Nuevo Reino de Granada. Comentario y mirada retrospectiva a los 60 años de su publicación
}

\author{
Quinas in the Flora from Royal Botanical Expedition of the New \\ Kingdom of Granada. A commentary 60 years after its publication
}

\author{
๑ José Luis Fernández-Alonso
}

Departamento de Biodiversidad y Conservación, Real Jardín Botánico-CSIC, Plaza de Murillo 2, 28014 Madrid, España.

\begin{abstract}
Resumen
Transcurridos ya 60 años de la publicación histórica de las quinas de la Flora de la Real Expedición Botánica del Nuevo Reino de Granada en el volumen 44 de esta obra, queremos presentar aquí un breve análisis retrospectivo sobre su génesis, contenido y alcances más relevantes. Este estudio pionero dio a conocer algunos manuscritos aun inéditos y el grueso de la incomparable iconografía de las quinas o cascarillas de la Expedición Botánica (17831816), grupo exquisitamente ilustrado y documentado en los actuales territorios de Colombia y Ecuador, bajo la dirección de José Celestino Mutis. Se comentan y revisan aquí estos aportes en el contexto del estudio taxonómico de este grupo de plantas en la América tropical y del desarrollo de su aplicación terapéutica.
\end{abstract}

Palabras clave: Cinchona, Ladenbergia, Cinchoneae, Colombia, Ecuador, Mutis, quinas, terapéutica.

\begin{abstract}
Sixty years after the historical publication of the quinas of the Flora of the Royal Botanical Expedition of the New Kingdom of Granada, in its volume 44, we want to present here a brief retrospective analysis on its genesis, content and scope. This pioneering study unveiled some unpublished manuscripts and the bulk of the incomparable iconography of the quinas or cascarillas of the Botanical Expedition (1783-1816), a group exquisitely illustrated and documented in nowadays territories of Colombia and Ecuador, under the direction of José Celestino Mutis. These contributions are discussed and reviewed here in the context of the taxonomic study of this group of plants in tropical America and their therapeutic application.
\end{abstract}

Key Words: Cinchona, Ladenbergia, Cinchoneae, Colombia, Ecuador, Mutis, quina trees, therapeutics.

\section{Introducción}

En el año 1957 fue publicado el volumen dedicado a las Quinas (género Cinchona L. s.l., Rubiaceae) de la Flora de la Real Expedición del Nuevo Reino de Granada, obra monumental en la que se empezaban a publicar los resultados de la Expedición desarrollada entre 1783 y 1816 en territorios que actualmente corresponden a Colombia y Ecuador, bajo la dirección de José Celestino Mutis. Transcurridos ya más de seis décadas desde la publicación histórica de las quinas de esta Expedición (Tomo 44 de esta Flora en gran formato), queremos mediante la presente revisión, mostrar algunos aspectos de la génesis, el contenido y los alcances más relevantes de esta publicación pionera sobre las quinas de la Expedición. La preparación de dicho tomo fue conducida y comentada en su día por dos científicos prominentes, el botánico colombiano Enrique Pérez-Arbeláez (1896-1972) y el médico y farmacólogo español Fernando Fernández de Soto Morales (1898-1966). En el tomo se dieron a conocer varios manuscritos históricos inéditos, el grueso de la iconografía de las quinas en La Expedición y varios capítulos obra de estos dos autores (Alejandre \& al., 2019).

Quinas de la Expedición, tomo 44 (1957). Se trata de una publicación en folio mayor o atlántico sobre las Cinchona L. y géneros afines (familia Rubiaceae) que aparte de los textos y los comentarios de los autores del tomo, incluye como materiales originales de la Real Expedición Botánica: sesenta y dos láminas -treinta y tres en color y

\footnotetext{
Correspondencia:

José Luis Fernández-Alonso; jlfernandeza@rjb.csic.es

Recibido: 8 de noviembre de 2019

Aceptado: 10 de noviembre de 2019

Editor: Elizabeth Castañeda
} 
veintinueve en negro-, tres facsímiles de manuscritos, dos mapas y un dibujo de nivelaciones barométricas. En el tomo se hace referencia a la autoría principal de José Celestino Mutis y Bosio y a los responsables de los comentarios y la revisión editorial de este tomo de la Quinología de la Real Expedición: Enrique Pérez-Arbeláez y Fernando Fernández de Soto Morales; del mismo modo se incluye el aporte de Francisco José de Caldas, y Francisco Antonio Zea (manuscritos) y de M. Romero y F.J Zabraraín y José María Carbonell (algunos dibujos y caligrafías). Contenido: [I]-XII, [1]-196 páginas, [62] h. de lám. col. y n. [Lam. 1-62]; $55 \mathrm{~cm}$.

Este es el tercer tomo editado (año 1957) de la Flora de la Real Expedición Botánica, una obra concebida en 51 volúmenes cuya publicación estaba a cargo de los Institutos de Cultura Hispánica de Madrid y Bogotá. Su edición había comenzado apenas 3 años antes con la publicación del primer tomo, con la Introducción histórica y plan de la obra a cargo de varios autores (Pérez-Arbeláez \& al, 1954). En la actualidad, los 39 tomos publicados de esta obra han sido reproducidos también en formato digital, gracias a un acuerdo de la Junta de Mutis (responsable de esta publicación) integrada por el Real Jardín Botánico, la Agencia Española de Cooperación Internacional para el Desarrollo, el Instituto Colombiano de Antropología e Historia y el Instituto de Ciencias Naturales de la Universidad Nacional de Colombia (AECID, Biblioteca Digital, 2019; RJBCSIC, Biblioteca Digital, 2019). Cabe mencionar también que la colección completa de láminas, diseños y disecciones (anatomías) de la Real Expedición Botánica (más de 6500 elementos), es accesible en alta resolución en la página web institucional del Real Jardín Botánico (RJB-CSIC).

Un gran prólogo para una gran noticia. El volumen 44 de la Flora de la Real Expedición Botánica del Nuevo Reino de Granada incluye los estudios sobre las quinas, realizados bajo la dirección de don José Celestino Mutis y los auspicios del rey Carlos III. En este se incluyen las láminas, los manuscritos y la información de herbario referentes a las quinas obtenidos durante dicha Expedición y que actualmente se conservan en el Real Jardín Botánico. Como prologan los autores, el de las quinas es un tomo particular que exige un tratamiento especial por algunos motivos sobresalientes, a saber: 1) La Real Expedición Botánica en el Virreinato se gestó en gran medida de la mano del estudio de las quinas, grupo vegetal que fuera profundamente documentado en diferentes aspectos (médicos, farmacológicos, fitogeográficos, sociológicos y comerciales), que rebasaban los típicamente botánicos y descriptivos propios de una Flora; 2) El trabajo exploratorio, iconográfico y descriptivo y la variada documentación obtenida y analizada sobre las quinas por el conjunto de los miembros de la Real Expedición, es sin duda la más copiosa y detallada entre los distintos grupos vegetales abordados (florones, orquídeas, canelos, guacos, bálsamos, mastrantos, cañas, bignonias, etc.) en el estudio de la flora neogranadina; 3) Los trabajos adelantados con las quinas llevaron aparejado un desarrollo comercial y estratégico importante en las provincias americanas, que fue objeto de numerosas disposiciones gubernamentales, litigios y consultas tanto en la Corte como en el virreinato, en los que inevitablemente se vio involucrado José Celestino Mutis y su Expedición así como los botánicos de la Flora de Perú, Hipólito Ruiz y José Pavón, (Gómez Ortega, 1779; Ruiz, 1792; Ruiz \& Pavón, 1801; Humboldt, 1822; Jaramillo, 1951); 4) A diferencia de lo que se hubiera esperado conseguir en general o resolver en un tratamiento taxonómico clásico para esta Flora, en el caso de las quinas y géneros afines de la familia del café (Rubiáceas) se encontraron importantes escollos que lo impidieron, por la multiplicidad de autores, criterios y procedencias de la información, que no pudo ser suficientemente cotejada en tiempos de la Expedición ni en fechas posteriores y tampoco en las previas a la edición y publicación del tomo 44 , como razonadamente justifican sus autores; 5) Se quiere ver también en el dispendio de tiempo, recursos y esfuerzos dedicados a las quinas a lo largo de todo el desarrollo de la Expedición Botánica, un factor importante que habría jugado en contra del avance normal y la finalización de la proyectada Flora de Bogotá; 6) Los autores puntualizan además, que al tratarse de un tema tan amplio e históricamente relevante, que rebasa el ámbito típicamente botánico, para la publicación del tomo se centraron en la presentación de los logros que Mutis y sus colaboradores obtuvieron durante la Expedición, más que en la redacción de una Quinología actualizada a la fecha, empresa sistemáticamente inabordable para los autores con los medios disponibles de consulta en la década de 1950.

De autores, edición e impresión. Enrique Pérez-Arbeláez, que fue fundador del Herbario Nacional Colombiano, director del Jardín Botánico José C. Mutis de Bogotá (Alejandre \& al., 2018), trata y redacta en el tomo lo relativo a la ambientación, a la historia y a la labor taxonómica de la Real Expedición en lo que a las quinas se refiere. Fernando Fernández de Soto Morales, en su día jefe de la Sección de Farmacología Experimental del Instituto José C. Mutis del Consejo Superior de Investigaciones Científicas de España, tuvo a su cargo las exposiciones y textos referentes a la parte farmacéutica y médica. El trabajo de presentación editorial y tipográfica de este tomo 44 (Figura 1A), así como la de los dos tomos anteriores, el histórico tomo 1 (Pérez-Arbeláez \& al., 1954) y el de las Pasifloráceas y Begoniáceas, tomo 27 (Uribe Uribe, 1955) es realización de Ediciones Cultura Hispánica y de los impresores Sucesores de Rivadeneyra, S. A., de Madrid, que pusieron a prueba en esta empresa las bondades de las artes gráficas españolas en la época con esta obra monumental abordada por los gobiernos de España y de Colombia. En la página de colofón del tomo, que reproducimos aquí (Figura 1B) se deja constancia de la fecha en que finalizó la impresión, en marzo de 1957, un mes después de que los restos mortales de Don José Celestino Mutis, fueron exhumados en la Iglesia de Santa Inés en Bogotá para ser depositados en el lugar donde reposan hoy, 
la capilla La Bordadita del Colegio Mayor Nuestra Señora del Rosario, escenario de muchas de sus contribuciones académicas (Díaz D., 1963; Hernández de Alba, 1963).

El abordaje de las quinas, sus circunstancias particulares y su relevancia. Reconociendo plenamente el mérito del primer uso de las cortezas febrífugas de los árboles de la quina a las comunidades ancestrales americanas, después de muchas pruebas y experiencias no siempre fructíferas y efectivas durante más de 150 años, los autores celebran el desarrollo que en tiempos recientes tuvo la ciencia de la Quinología y la demostración de sus efectos farmacológicos incontestables. Se resalta también la importancia que tuvo para el estudio y comercio de la quinas, la ciudad actualmente ecuatoriana de Loja, como primer centro de abastecimiento de este remedio en la América hispana, empeño al que tantos esfuerzos dedicó Mutis. En palabras también de los autores. "La preciosidad con que la Expedición, fiel a las normas de su ya difunto director, preparaba para el Rey los manuscritos relativos a los árboles de quina

A.

TOMO CUARENTA Y CUATRO

\section{QUINAS DE LA REAL EXPEDICION BOTANICA DEL NUEVO REINO DE GRANADA}

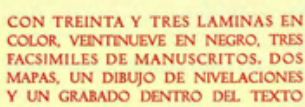

CON TREINTA Y TRES LAMINAS EN COLOR, VEINTINUEVE EN NEGRO, TRES FACSIMILES DE MANUSCRITOS. DOS YAPAS, UN DIBUJO DE NIVELACIONES

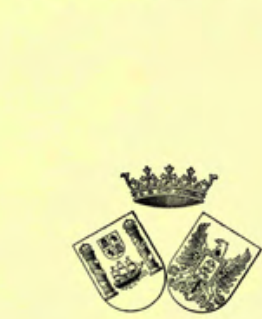
MADRID
igs?

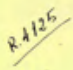

del Nuevo Reino, nos aconseja la reproducción de los originales revisados por Sinforoso Mutis, y copiados por la pluma elegantísima de José María Carbonell... Esta obra completa es indispensable a quienes aspiren a resolver los problemas históricos, médicos y taxonómicos de las quinas oficinales" [Pérez-Arbeláez \& Fernández de Soto, 1957]. Hacen referencia también a las variadas fuentes de información a las que han necesitado acudir para articular las diferentes referencias a las quinas de la Nueva Granada y sus usos, como complemento de la fuente primaria del Archivo de la Expedición (Madrid). Una especial mención se hace a don Francisco de las Barras y Aragón y a don Luis Ángel Arango por los datos facilitados de los Archivos de Indias en Sevilla y del Banco de la República en Bogotá. Celebran también que la publicación de esta nueva entrega contribuya a la consolidación del recién iniciado proyecto binacional empeñado en publicar esta Flora de Mutis (Jaramillo, 1953; Pérez-Arbeláez \& al., 1954).

B.
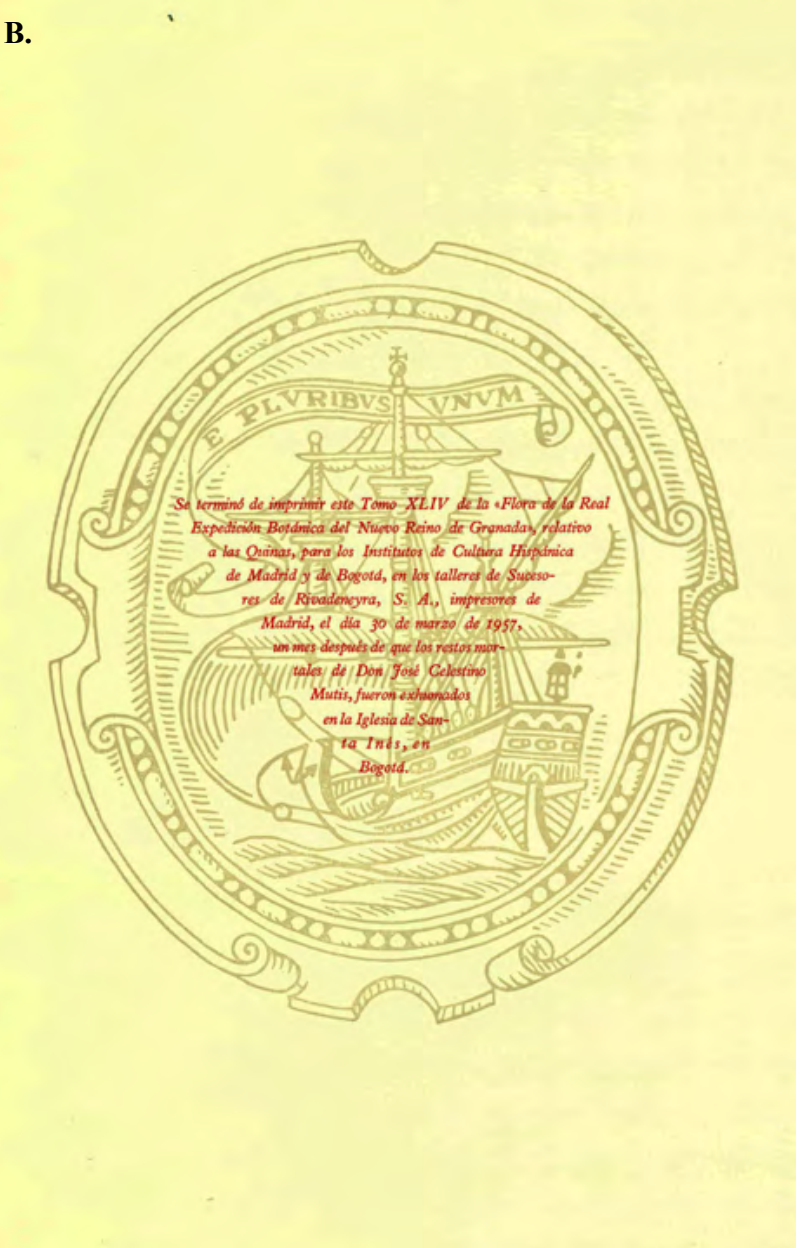

Figura 1. A (Izquiera). Portadilla del tomo 44, Quinas de la Real Expedición Botánica del Nuevo Reino de Granada, publicado en 1957. B (Derecha). Colofón del Tomo donde se deja constancia de los detalles de la impresión y se informa de la efeméride coincidente de la exhumación de los restos mortales de Don José Celestino Mutis en la Iglesia de Santa Inés en Bogotá, siendo su destino posterior, la capilla La Bordadita del Colegio Mayor Nuestra Señora del Rosario. 


\section{Sobre el tomo 44 de la Flora de Mutis}

Parte I. Historia de las quinas de la Expedición e ideas sobre su terapéutica y proyectado beneficio. En los Capítulos I a III Pérez-Arbeláez nos presenta una descripción histórica de las quinas de la Expedición Botánica acompañada de una cronología de la labor adelantada en este campo. En ella se describen los ambientes, las condiciones y las actividades que desarrolló Mutis y su Expedición, encaminadas a documentar y dar a conocer los valiosos árboles de la corteza febrífuga, tanto en el Virreinato como en la Corte de Madrid, entre 1761 y 1809. Le sigue una transcripción del valioso documento elaborado por Mutis: Real proyecto del Estanco de quina y sus establecimientos y del Apéndice de la Relación informativa práctica...de Miguel de Santiesteban. El documento preparado por don José Celestino Mutis para el Gobierno de Madrid (Mutis, 1786) contiene un planeamiento y una completa prospección de las explotaciones de quina incluyendo aspectos comerciales. Este documento ha sido considerado pionero también en la sistematización detallada de un tema forestal en América, que incluía la organización operativa en las exportaciones de vegetales americanos hacia los centros de consumo y distribución en España y el continente europeo (Hernández de Alba, 1991). En el Capítulo IV - Ideas sobre Taxonomía de las quinas de la Real Expedición Botánica en general a cargo de E. Pérez Arbeláez, se analizan los principios e ideas taxonómicas que sobre quinas siguió la Real Expedición, con numerosos comentarios en castellano añadidos a las descripciones latinas singulares de cada uno de los dibujos reproducidos, en concordancia con el plan taxonómico de esta Flora. En el Capítulo $\boldsymbol{V}$ - Ideas de don José C. Mutis sobre farmacología y terapéutica de las quinas, se aporta con carácter especial en este tomo, abundante información para la evaluación de la labor científica de Mutis en el campo de la farmacología y terapéutica vista desde la ciencia de mitad del siglo XX es el trabajo a cargo de Fernández de Soto (1957: 75-95).

Parte II. Historia de los árboles de la quina y otros escritos quinológicos de la Real Expedición Botánica. En primer lugar, se presentan dos publicaciones del siglo XIX debidas a miembros de la Expedición Botánica, ambas acompañadas de la correspondiente introducción de E. Pérez-Arbeláez: A) Memoria sobre la quina, según los principios del señor Mutis, escrita en 1800 por don F. A. Zea. y B) Memoria sobre el estado de las quinas en general y, en particular sobre las de Loja, manuscrito de 1805 debido a don F. J. de Caldas. Estos dos estudios sobre quinas de F. A. Zea, y la de F. J. de Caldas, representan el hilo conductor de las ideas de J. C. Mutis (Fernández Pérez \& al., 2004; Amaya \& Torres, 2016) y a la vez incluyen muchas apreciaciones y matices particulares de sus autores, asociados a los escenarios en que cada uno de ellos hubo de actuar en las controversias surgidas que se relacionaban con las quinas.
En segundo lugar, se publica la "Historia de los Arboles de Quina, obra póstuma del doctor don José C. Mutis, concluida y arreglada por don Sinforoso Mutis Consuegra" con comentarios en la parte sistemática por E. Pérez Arbeláez. Este capítulo integra la Historia de los Arboles de Quina, escrita por J. C. Mutis y con el discurso preliminar y la parte sistemática complementada por su sobrino Sinforoso Mutis, a quien, después de la muerte del primero, quedó confiada la parte botánica de la Expedición y la continuación de la Flora de Bogotá. Esta parte va ilustrada con 62 láminas, que representan solo una fracción de la magnífica iconografía de la Real Expedición Botánica dedicada a los árboles de la quina. Considerando las láminas de quinas del catálogo general del Archivo Mutis (San Pio, 2008) que no fueron incluidas en el tomo 44 y el hecho de que algunas más fueron obsequiadas a Linneo y Humboldt en tiempos de Mutis, como consta en los documentos (Amaya, 2015), podemos tener una idea de la minuciosidad con que se rastreó e iconografió este grupo de plantas. El plan de estudio de esta Quinología de Mutis fue sumamente exhaustivo y por lo general nos dejó cuatro láminas distintas de cada una de las formas o variantes diferentes, que catalogaron como pertenecientes al género Cinchona: $\mathrm{a}-\mathrm{b})$ una en color con la inflorescencia y las anatomías de la flor, con un calco de la misma en negro; y c-d) otra en color con la foliación, la infrutescencia y su disección, con una cuarta como calco en negro de la anterior. Cada variante o morfotipo representado lleva un número de orden diferente (en numeración romana) y las distintas láminas de las partes representadas en color y en negro van numeradas con letras arábigas complementarias (a,b,c,d.). Los autores se felicitaban por la publicación completa de la iconografía de las quinas a pesar de algunas vicisitudes y traslados sufridos por la iconografía original, felizmente superadas con celo y cuidado de los directores de turno. Hasta la fecha de publicación del tomo, fueron varios los autores e importantes los análisis y estudios publicados que en alguna medida citaron o hicieron referencia a los documentos quinológicos de la Real Expedición Botánica dirigida por don José C. Mutis y continuada por Sinforoso Mutis: Karsten (1858-1861), Howard (1862), Triana (1872), Standley (1930, 1936), Osorio (1945) y Fosberg (1951) entre otros, trataron sobre quinas procedentes de lo que fue el Nuevo Reino de Granada y analizaron la labor de Mutis y su escuela. Sin embargo, quedaban aun muchos puntos obscuros sobre el desarrollo de esta Flora que los autores del tomo logran abordar y documentar para tal ocasión.

\section{Algunos comentarios sobre la taxonomía de los icones del Tomo 44 de la Flora.}

\section{A-Aportes de Enrique Pérez-Arbeláez.}

Bajo el título "Ideas sobre taxonomía de las quinas de la Real Expedición Botánica en general”, Pérez-Arbeláez enmarca con precisión el trabajo taxonómico adelantado con las quinas por la Expedición haciendo referencia a los nombres vernáculos y a los caracteres diferenciales 
manejados por Mutis, y lo coteja después con los tratamientos y clasificaciones posteriores a la Expedición y con las vigentes en la década de 1950 en que se publicó el tomo (Pérez-Arbeláez, 1957: 67-74). Tiene en cuenta en especial los tratamientos recientes de las Cinchoneae de P. C. Standley para algunas floras del norte de Sudamérica, en los que se restringe la circunscripción del género Cinchona y se ubican o transfieren muchas de las especies originales de Cinchona en otros géneros reconocidos dentro de esta tribu (Standley 1930, 1931, 1936). Del mismo modo, muchos de los nombres de especies descritas por otros autores dentro del género Cinchona, son catalogados como sinónimos de especies ya nombradas, siguiendo el criterio de varios botánicos e investigadores de la época que trabajaron o trataron el grupo (Steere, 1944; Osorio, 1945; Little, 1947; Camp, 1949, 1952; Hodge, 1948; Jaramillo, 1951; Fosberg, 1951). No obstante, sin ser el objeto de este escrito, cabe precisar aquí que el género Cinchona en su circunscripción actual agrupa poco más de 20 especies con distribución neotropical restringida a gradientes montañosos, principalmente en la zona andina (Anderson, 1998; Anderson \& Antonelli, 2005). Con base en análisis moleculares recientes se considera que Cinchona constituye la tribu monofilética Cinchoneae, junto con Ladenbergia y siete géneros más. (Anderson \& Antonelli, 2005; Maldonado \& al., 2017). Aparte de Cinchona y Ladenbergia, los otros tres géneros de Rubiáceas que formaban parte de las quinas de la Expedición, y que han sido reubicados posteriormente en tribus diferentes de acuerdo con los nuevos análisis morfológicos y moleculares son: Macrocnemum $\mathrm{P}$. Br. y Ferdinandusa Phol. en la tribu Condamineae y Cosmibuena Ruiz \& Pav. en la Tribu Hillieae S.P. Darwin. (Anderson, 1995; Anderson \& Antonelli, 2005).

Sobre la identificación de las láminas originales y descripciones del manuscrito de la "Historia de los árboles de la quina", Pérez-Arbeláez hace el siguiente comentario: "El análisis comparativo entre las labores sistemáticas quinológicas de J. C. Mutis, de F. J. Caldas, de S. Mutis y de J. J. Triana, de Zea, de Howard, de Karsten y de Humboldt y Bonpland nos servirá para dar por terminado nuestro modesto cometido". (Perez-Arbeláez, 1957).

a) Rubiáceas Cinchonoideas. En primera instancia y después de hacer referencia al encuadre sistemático del orden Rubiales en el sistema de A. Engler y de la familia de la Rubiáceas y sus subdivisiones, Pérez-Arbeláez enmarca las siete especies de Cinchona contempladas por Mutis en el contexto de los diferentes géneros reconocidos en el tratamiento de las Rubiáceas Cinchonoideas de Standley (1930, 1936). El arreglo sistemático resultante fue el siguiente:

1-Cinchona L. dos de ellas (Cinchona lanceifolia Mutis y C. cordifolia Mutis = Cinchona pubescens Vahl. (Figura 2 A-B) se mantienen en el género linneano original, en tanto
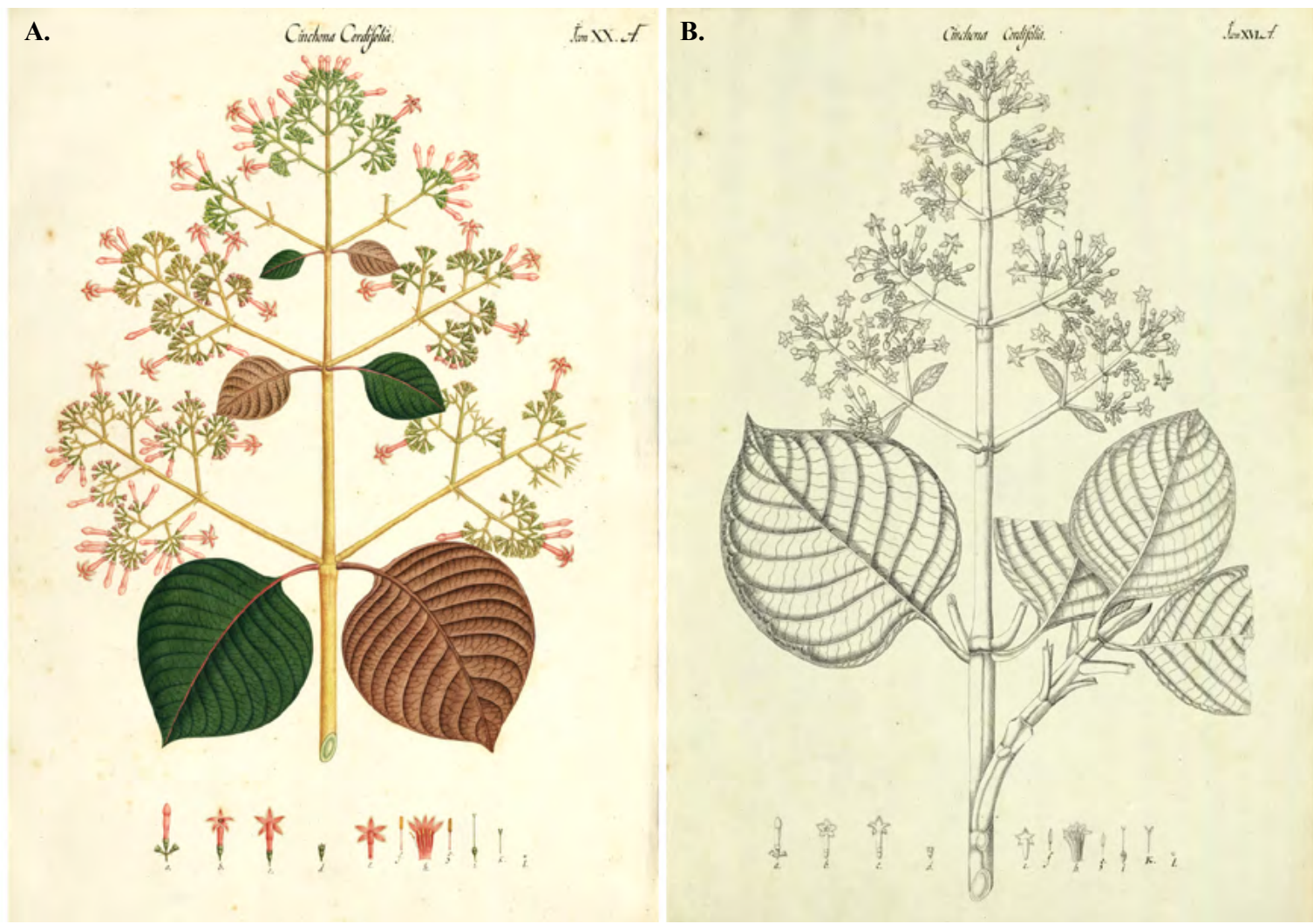

Figure 2 A-B. Cinchona cordifolia Mutis = Cinchona pubescens Vahl A- Lámina 2867- Rama florida y disección de las flores (color). B- 2863a- Rama florida y disección de las flores (sepia) Láminas: CReal Jardín Botánico-CSIC. 
que las otras cinco quinas de Mutis quedan formalmente ubicadas en los siguientes cuatro géneros, hoy pertenecientes a diferentes tribus:

2-Ladenbergia Klotzch. in Hayne (Cinchona oblongifolia Mutis = Ladenbergia oblongifolia (Humb. ex Mutis.) L. Anderson (Figure 3 A-B) y C. ovalifolia Mutis = Ladenbergia macrocarpa (Vahl.) Klotzch.) (Figure 3 C-D).

3-Macrocnemum P. Br. (Cinchona parviflora Mutis = Macrocnemum parviflorum (Mutis)Triana", afin a Macrocnemum roseum (Ruiz \& Pav.) Weddell) (Figura 4 A-B, C-D).

4-Ferdinandusa Pohl. (Cinchona dissimiliflora Mutis = Ferdinandusa dissimiliflora (Mutis ex Humb.) Stand (Figura 5. A-B).

5-Cosmibuena Ruiz. \& Pav. (Cinchona longiflora Mutis = Cosmibuena grandiflora (Ruiz \& Pav.) Rusby).

b) Mas quinas y nombres de Triana. En segundo lugar hace referencia a la existencia en Colombia de otras cuatro especies de Cinchona (Pérez-Arbelaez, 1957: 7273), ya recogidas en la reciente obra de Standley, que no se encontraban en los documentos de la Expedición (Cinchona pitayensis Wedd. (descrita en 1849), C. barbacoensis $\mathrm{H}$. Karst. (d. en 1859), C. henkleana H. Karst. (d. en 1859) y $C$. rosulenta Howard ex Wedd. (d. en 1869). También presenta una detallada relación de las identificaciones dadas por J. J. Triana a los icones de la Historia de los árboles de la Quina de la Expedición (Triana, 1872), alertando sobre la corrección de algunas epigrafías que no aparecieron bien identificadas en la transcripción de la obra publicada con posterioridad en la Revista de la Academia Colombiana de Ciencias (Triana, 1937, 1938a, 1938b). En esta compleja relación de nombres y variedades que recoge Pérez-Arbeláez, vemos que Triana procede de la siguiente manera:

i) Mantiene los nombres de Mutis para las especies Cinchona lancifolia y C. cordifolia (especies en las que reconoce un total de 12 taxones varietales diferentes, asociados a nombres previamente asignados a plantas de este género por H. Ruiz, J. Pavón, Tafalla y Lambert entre otros.

ii) Reconoce cinco nombres específicos diferentes a los de Mutis para las otras cinco especies de quina de Mutis, en los que ya aparece la inclusión de algunas de estas quinas en los géneros cercanos (Cosmibuena, Macrocnemum).

c) Identificaciones de Pérez-Arbeláez. Por último, incluye una tabla en la que se presentan en palabras de Pérez-Arbeláez "las equivalencias a que nosotros mismos llegamos, entre los icones de la quinología y los materiales así descriptivos como de herbario que nos han servido para comentarla". En este punto cabe resaltar en relación con lo ya indicado de las siete especies de quinas de Mutis, que dos se siguen manteniendo en el género Cinchona y las otras cinco son tratadas asimismo en los cuatro géneros de Cinchoneae ya mencionados. Cabe anotar dos detalles particulares.

i) En el tomo se trata a Cinchona lancifolia Mutis como sinónimo de C. officinalis L., siguiendo el criterio de la época. ii) Entre los diferentes taxones varietales que reconoce en las especies tratadas, sugiere, además, y en modo condicional (Pérez-Arbeláez, 1957: 73), tres nombres nuevos de variedades en Cinchona officinalis L. que son los siguientes: "almizclillo P. Arb.", “alausi P. Arb." y "saraguro P. Arb.", asociados a otros tantos icones de la Cinchona lancifolia Mutis de la Quinología original. Estas categorías infraespecíficas, que no son propuestas formalmente en esta obra -como aclara su autor- no reunían por ello los requisitos de validez de nombres que establece el código de nomenclatura botánica (Thurland \& al., 2018), en su artículo 36.1. Sobre este caso dice de forma explícita: "Un nombre no está válidamente publicado (a) cuando no es aceptado por el autor en la publicación original; (b) cuando fue propuesto como "nombre provisional", es decir, meramente en anticipación de la aceptación futura del taxón en cuestión, o de una circunscripción, posición o rango particular del taxón".

\section{B-Algunas referencias posteriores a la publicación.}

Como bien dice Pérez-Arbeláez (1998 [1 ${ }^{\text {a }}$ ed.1967]), una década después de haberse empleado a fondo en la publicación del tomo 44 de las quinas de la Flora de Mutis, "La historia de la quina, o mejor, de los quinos, impregna todos los periodos de la vida de la Expedición Botánica y aun los rebasa". Y al referirse a la primera obra quinológica de Mutis (1793) comenta "Tuvo razón Don José Celestino para titular su Quinología: El Arcano de la Quina, título que evoca una grande y oscura problemática, en cuya declaración el era, en razón del puesto que se había buscado, el único arriesgado precursor". Hernández de Alba (1991) en su obra de las quinas amargas analiza con cierto detalle algunos aspectos relacionados con el quehacer de Mutis y su Expedición en el estudio de este grupo de plantas y en la polémica existente en la época, en relación con su administración y el problemático comercio de este recurso extractivo. Díaz P. (2003) presenta un escueto pero certero análisis sobre el estudio de las quinas de Colombia por parte de José Celestino Mutis, en el que hace referencia a la primicia y originalidad del "Arcano de la quina" y también a las contribuciones de Francisco José de Caldas (1805, 1966) y de .J.J. Triana (1872) al estudio de las quinas neogranadinas.

Otros aspectos sobre el trabajo de J.C. Mutis, A. Zea, F.J. de Caldas y Sinforoso Mutis con las quinas de la Nueva Granada, han sido comentados por diferentes autores en tiempos recientes (Díaz P., 1992; Appel, 1994; Gutiérrez, 1995; Fernández Pérez \& al, 2004; Amaya \& Rendón-Acosta, 2016; Cuvi, 2018; Fernández-Alonso, 2019). Del mismo modo, en Mantilla \& Díaz P. (1992) y en Díaz P. (1993 y 2003) se hace una justa mención del aporte de Fray Diego García, adjunto de la Expedición, al estudio de las quinas de la Expedición. Fray Diego envió muestras de cortezas y partes florales de especies de varias regiones de Colombia que no habían sido visitadas por otros colaboradores de la Expedición, muestras que actualmente 


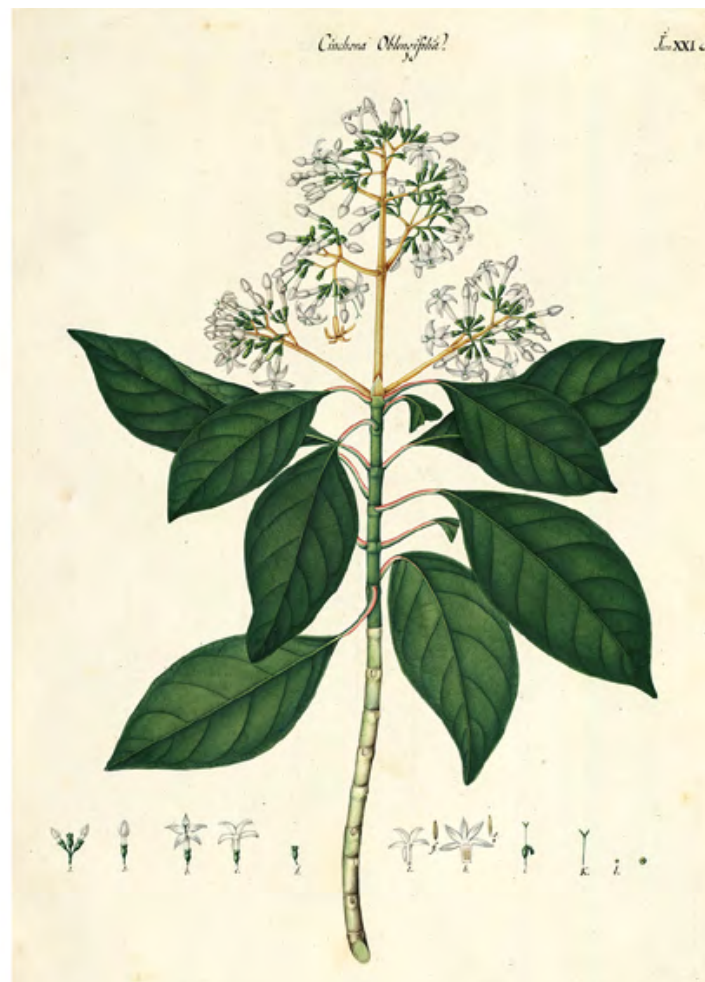

A.
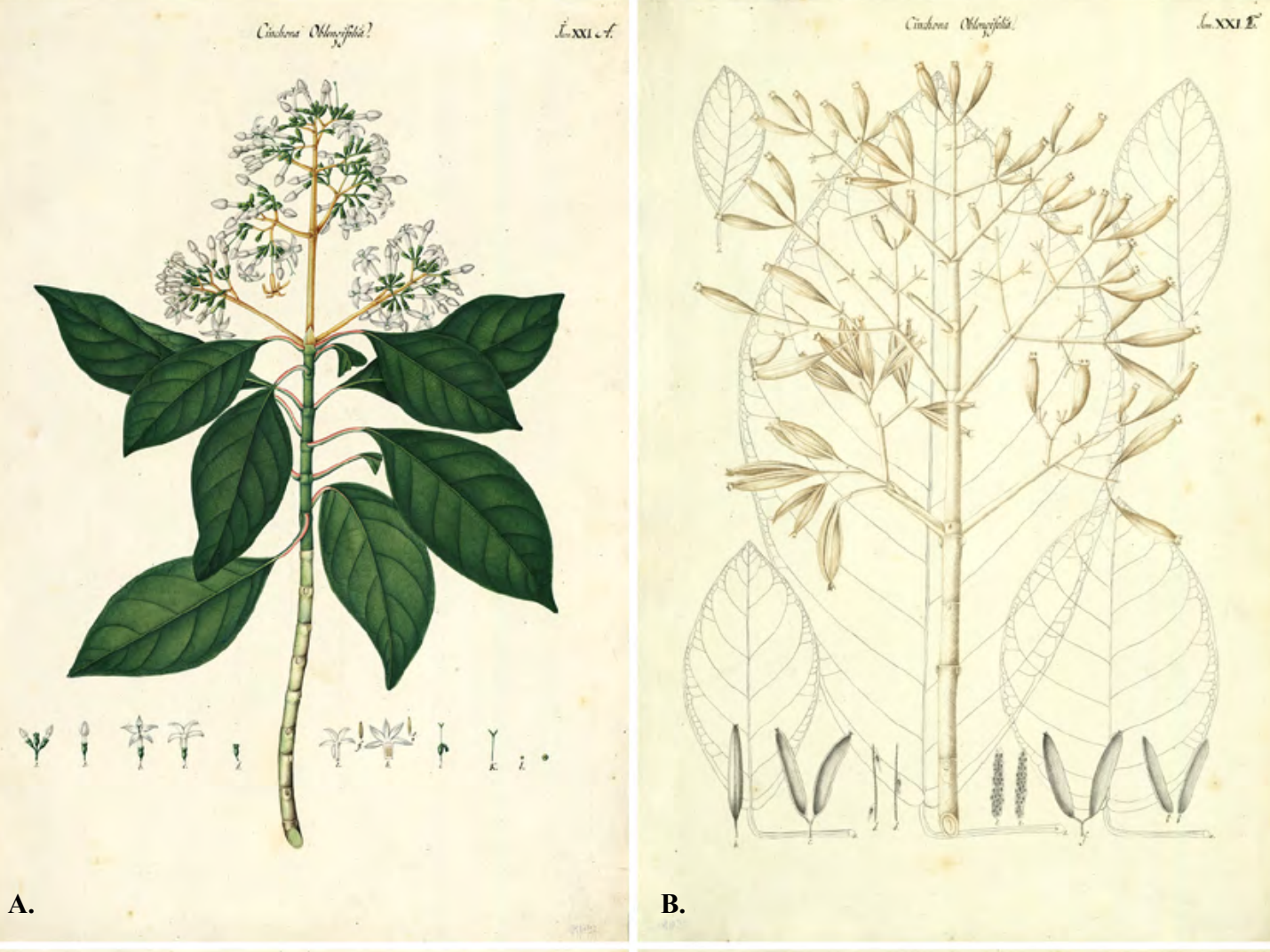

B.
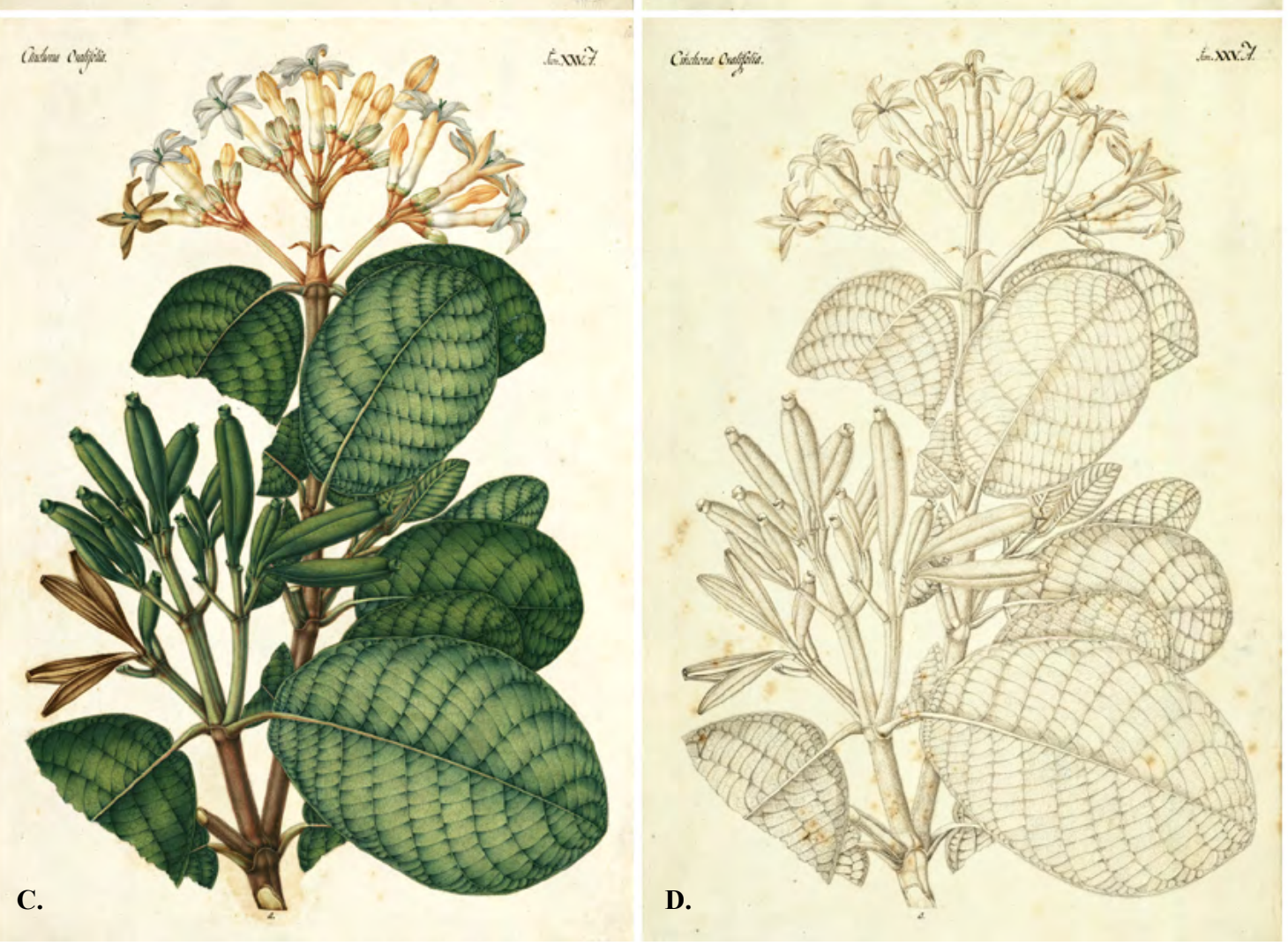

Figura 3. A-B. Cinchona oblongifolia Mutis = Ladenbergia oblongifolia (Humb. ex Mutis.) L. Anderson. A- Lámina 2869- Rama florida con disección de las flores (color) alineada en su base. B- Lámina 2869c- Infrutescencias, representación esquemática de las hojas y disección de los frutos (sepia). C-D. Cinchona ovalifolia Mutis = Ladenbergia macrocarpa (Vahl.) Klotzch.; C- Lámina 2873- Rama florida finamente coloreada. D- Lámina 2873a- Copia de la rama florida (sepia). Láminas: CReal Jardín Botánico-CSIC 


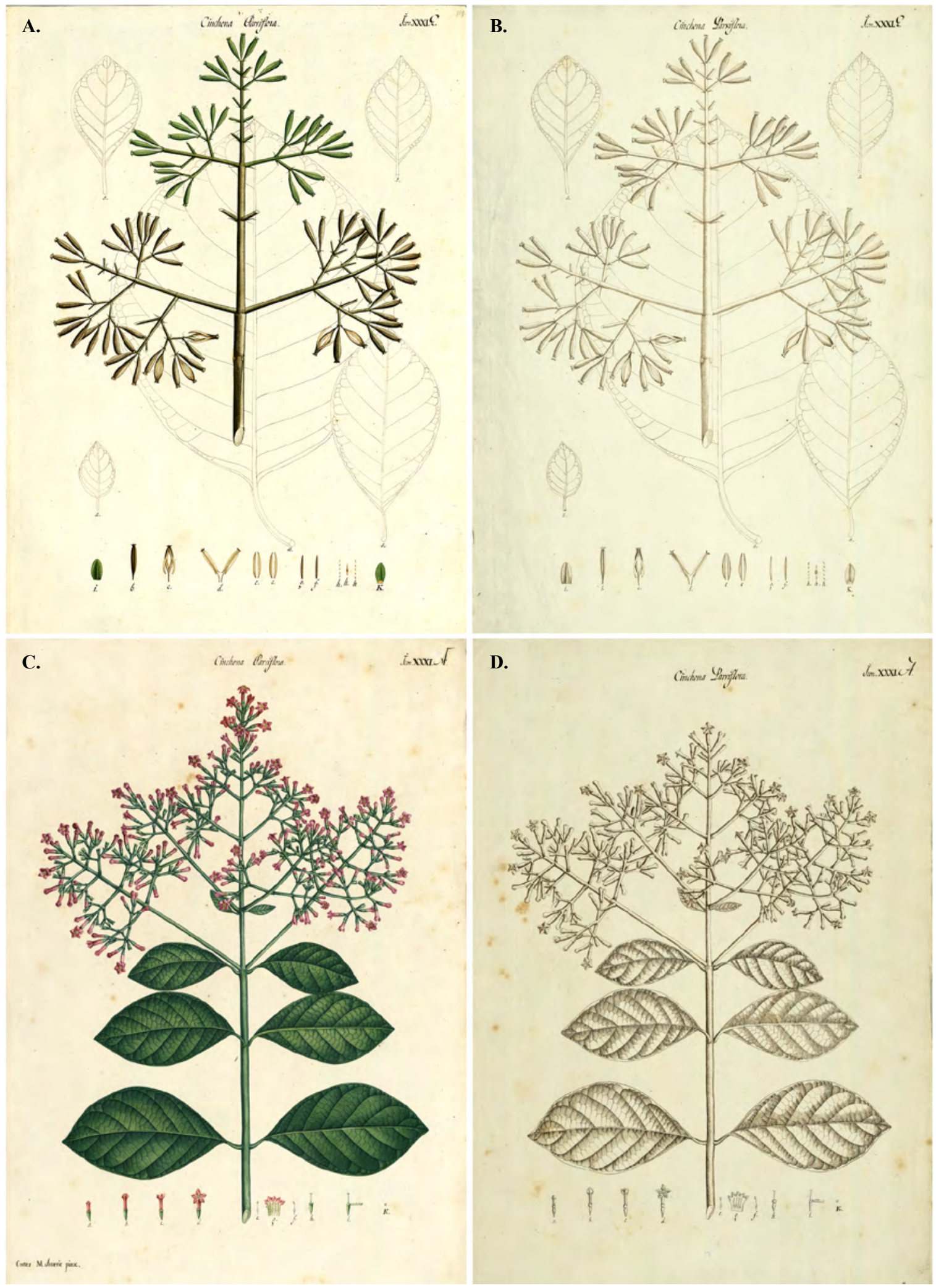

Figure 4 A-B. Cinchona parviflora Mutis = afin a Macrocnemum roseum (Ruiz \& Pav.) Weddell). A- Lámina 2879b- Infrutescencias, representación esquemática de las hojas, estipulas y disección de los frutos (color). B- Lámina 2879c- Infrutescencias, representación esquemática de las hojas, estipulas y disección de los frutos (sepia). C-D. Cinchona parviflora Mutis = afin a Macrocnemum roseum (Ruiz \& Pav.) Weddell). C- Lámina 2879- Rama florida y disección de las flores (color). D- Lámina 2879a- Rama florida y disección de las flores (sepia). Láminas: CReal Jardín Botánico-CSIC 

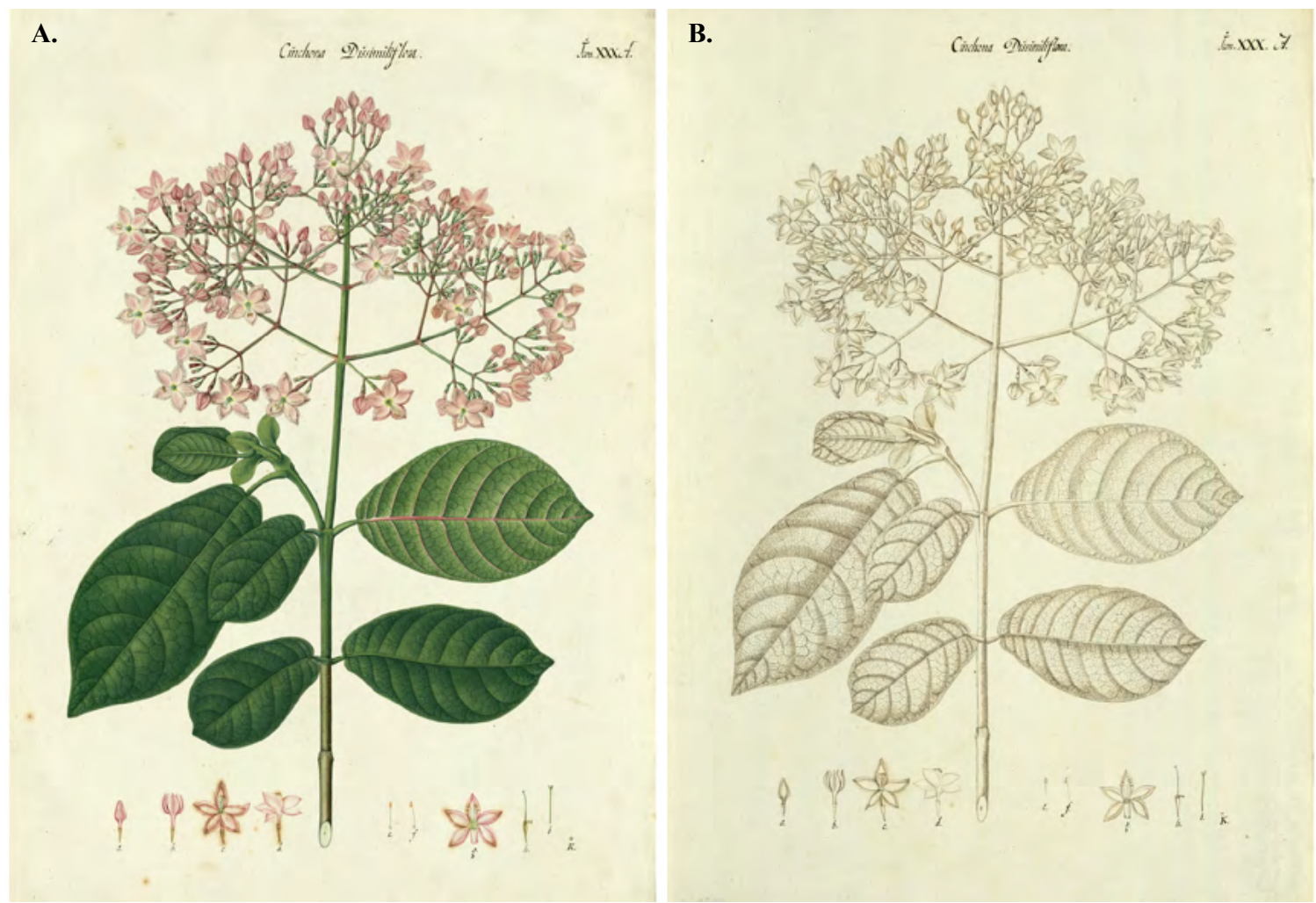

Figura 5. A-B. Cinchona dissimiliflora Mutis = Ferdinandusa dissimiliflora (Mutis ex Humb.) Stand. A- Lámina 2878- Rama florida y disección de las flores (color). B- Lámina 2878a- Rama florida y disección de las flores (sepia). Láminas: CReal Jardín Botánico-CSIC

se conservan catalogadas en el herbario y en la carpoteca de Mutis en el Real Jardín Botánico (Fernández-Alonso \& L. Suescun, 2019).

\section{A modo de conclusión}

Sin haber entrado a hacer nuevas precisiones taxonómicas sobre las especies de quinas representadas en las láminas y herbarios de la Real Expedición Botánica del Nuevo Reino de Granada, que no era el objetivo de este trabajo, al llegar a este punto, esperamos no obstante haber conseguido nuestro verdadero propósito. No es otro que presentar y discutir con algún detalle la génesis, el contenido y los alcances del tomo 44 de la Flora de Mutis, publicado en 1957 y dedicado enteramente al estudio histórico de los árboles de la quina, a la luz del conocimiento sistemático que de éste grupo de plantas tenemos en la actualidad. En este tomo se muestra sin duda, como en ningún otro, la impronta del trabajo perfeccionista de José Celestino Mutis, que nunca daba un tema por concluido, precisamente en el estudio del grupo de plantas que le fuera encomendado con especial prioridad por la Corona y que el abordó con mayor ahínco y dedicación.

\section{Agradecimientos}

Al Real Jardín Botánico (Biblioteca, Archivo y Herbario) y al Consejo Superior de Investigaciones Científicas las facilidades dadas para el desarrollo de este trabajo. A los colegas de la Academia Colombiana de Ciencias, Alberto
Gómez Gutiérrez y Elizabeth Castañeda, por toda su colaboración durante la escritura y revisión de este documento. Asimismo, al colega Ramón Morales, que guarda lazos de parentesco con uno de los autores del tomo, el médico y farmacólogo don Fernando Fernández de Soto Morales, por la amable revisión de este manuscrito.

\section{Referencias}

AECID. Agencia Española de Cooperación Internacional para el Desarrollo. Biblioteca digital. Madrid. (2019). http:// bibliotecadigital.aecid.es/bibliodig/es/catalogo_imagenes/ grupo.cmd?path $=1008460$

Alejandre, J.A., Benito Ayuso, J., Fernández-Alonso, J.L., García-López, J.M., Mateo Sanz, G. (2018). Notas sobre las colecciones burgalesas y otras plantas europeas de Enrique Pérez-Arbeláez (1896-1972), depositadas en el Herbario Nacional Colombiano (COL). Fl. Montiberica. 70: 3-49. (III-1918).

Amaya, J.A. (2015). "Como débil muestra de admiración y gratitud". José Celestino Mutis en la obra de Humboldt y Bonpland. Estudio preliminar”. Pp 76-100, en: Restrepo, O. (ed.) Ensamblado en Colombia I. Bogotá.

Amaya, J.A., Rendón Acosta, J.L. (2016). La formación de Hebephilo naturalista, 1791-1794. Fronteras de la Historia. 21 (2): 104-131.

Amaya, J.A., Torres Moreno, J.V. (2016). Ciencia y economía en el Nuevo Reino de Granada. Las comisiones de Sebastián José López Ruiz, 1778-1803. Historia Caribe. 11 (29): 183-213. 
Andersson, L. (1995). Tribes and genera of the Cinchoneae complex (Rubiaceae). Ann. Missouri Bot. Gard. 82: 409-427.

Andersson, L. (1998). A revision of the genus Cinchona (RubiaceaeCinchoneae). Mem. New York Bot. Gard. 80: 1-75.

Andersson, L. \& Antonelli, A. (2005). Phylogeny of the tribe Cinchoneae (Rubiaceae), its position in Cinchonoideae, and description of a new genus, Ciliosemina. Taxon. 54 (1): 17-28.

Appel, J.W. (1994). Francisco José de Caldas: A scientist at Work in Nueva Granada. Trans. Amer. Philos. Soc., New Ser. 84 (5): 1-154.

Caldas, Francisco J. (1805). "Memoria sobre el estado de las Quinas en general y en particular sobre las de Loxa". Archivo del Real Jardín Botánico, sección Mutis.

Caldas, Francisco J. (1966), "Memoria sobre el estado de las quinas en general y en particular sobre la de Loja [1805]". Pp. 241260. En: Obras completas de Francisco José de Caldas: publicadas por la Universidad Nacional de Colombia como homenaje con motivo del sesquicentenario de su muerte 1816 - Octubre 29 -1966, Bogotá, Imprenta Nacional.

Camp, W.H. (1949). Cinchona at high altitudes in Ecuador. Brittonia. 6: 394-430.

Camp, W.H. (1952). Plant hunting in Ecuador. Mem. New York Bot. Gard. 8 (1): 1-24.

Cuvi, N. (2018). Tecnociencia y colonialismo en la historia de las Cinchona. Asclepio. 70 (1): 1-13.

Díaz Diaz, O. (1963). Acta suscrita en la catedral primada para hacer entrega de los restos del sabio Mutis al Colegio Mayor Nuestra Señora del Rosario. Rev. Coleg. May. Ntra. Sra. del Rosario, Bogotá. 2 (463-464): 181-182

Díaz Piedrahita, S. (1992). Francisco José de Caldas y la botánica. Rev. Acad. Colomb. Ci. Exact. 18 (70): 269-382.

Díaz Piedrahita, S. (1993). Perfil científico de dos comisionados de la Expedición Botánica. Senderos. 5 (25-26): 564-575.

Díaz Piedrahita, S. (2003). Las quinas en el mundo y en Colombia. Rev. Medicina. 25 (2): 128-130.

Fernández-Alonso, J.L. (2019) Las plantas de Francisco José de Caldas y su contribución a los herbarios y escritos de la Flora de Bogotá. Pp.251-266. En: Álvarez, Y., Díez, C.A, Moreno, A. \& I.F. Suarez (eds.) Bicentenario Francisco José de Caldas 1768-1816. Editorial Universidad del Rosario. Bogotá.

Fernández-Alonso, J.L. \& Suescun Florez, L. (2019). La catalogación e identificación taxonómica de las muestras de la Carpoteca de Mutis en el Real Jardín Botánico de Madrid. Memorias X Congreso Colombiano de Botánica.Simposio de colecciones. 18-22 agosto 2019 - Florencia, Caquetá, Colombia. Asociación Colombiana de Botánica.

Fernández de Soto M., F. (1957). "Capitulo V. Ideas de don José C. Mutis sobre farmacología y terapéutica de las quinas". Pp. 75-95, en: Pérez-Arbeláez, E., Fernández de Soto Morales, F. (Eds.). Quinas de la Real Expedición Botánica del Nuevo Reino de Granada. Flora de la Real Expedición Botánica del Nuevo Reino de Granada Vol. 44. Instituto de Cultura Hispánica. Madrid.

Fernández Pérez, J., Jiménez Artacho, C., Fonfría Díaz, J. (2004). "Las quinas de Caldas". Actas VIII Congreso de la Sociedad Española de Historia de las Ciencias y de las Técnicas. Logroño: Universidad de la Rioja. Pp.559-583.

Fosberg, F.R. (1951). Temprana historia de la quina. Rev. Acad. Colomb. Ci. Exact. 18 (30): 273-274.
Gómez Ortega, C. (1779). Instrucción sobre el modo más seguro y económico de transportar plantas vivas.... Madrid: Joachin Ibarra. (Madrid: Biblioteca de clásicos de la farmacia española, 1992), pp. 37-38.

Gutiérrez Ramos, J. (1995). Sinforoso Mutis. Su vida y su obra. Fondo FEN Colombia. 120 pp.

Hernandez de Alba, G. (1963). El sabio Mutis, Colegial Rosarista: un capítulo de nuestra gloriosa crónica. Rev. Colegio Mayor Nuestra Señora del Rosario, Bogotá. 72 (463-464): 201-205.

Hernández de Alba, G. (1991). Quinas amargas. El sabio Mutis y la discusión naturalista del siglo XVIII. Academia de Historia de Bogotá. Tercer Mundo Editores. Colombia. $254 \mathrm{pp}$

Hodge, W.E. (1948). Wartime Cinchona procurement in Latin America. Econ. Bot. 2: 229-257.

Howard, J.E. (1862). Illustrations of the Nueva Quinologia of Pavon, with coloured plates by W. Fitch, F.L.S., and observations of the Barks described. London, Lovell Reeve \& Co.

Humboldt, A. von. (1821), Account of the Cinchona Forests of South America. Pp. 19-59. En: An illustration of the genus Cinchona: comprising descriptions... Londres, Print. J. Searle.

Jaramillo, A.J. (1951). Estudio crítico acerca de los hechos básicos en la historia de la quina. Rev. Acad. Colomb. Ci. Exact. 8 (30): 245-272.

Jaramillo A., J. (1953). Don José Celestino Mutis y las expediciones botánicas españolas del siglo XVIII al Nuevo Mundo. Rev. Acad. Colomb. Ci. Exact. 9 (33-34): 14-31.

Karsten, G.H. (1858-861). Florae Columbiae... Vol 1. Dummler, Berlin.

Little, R.R. (1947). Histology of barks of Cinchona and some related genera occurring in Colombia. Rev. Acad. Colomb. Ci. Exact. 7 (27): 404-425.

Maldonado, C., Persoon, C., Alban, J., Antonelli, A. \& Ronsted, N. (2017). Cinchona andersonii (Rubiaceae), a new overlooked species from Bolivia. Phytotaxa. 297 (2): 203-208.

Mantilla, L.C. \& Díaz Piedrahita, S. (1992). Fray Diego García, su vida y su obra científica en la expedixión Botánica. Colecc. Enrique Pérez-Arbeláez 7. Academia Colombiana de Ciencias Exactas, Físicas y Naturales. Bogotá. 284 pp.

Mutis, J.C. (1786). "Diciembre 20. Informe de Mutis a S. M. el rey don Carlos III sobre la explotación, estanco y comercio de la quina". Está concluido en tal fecha, pero se firma y se despacha el 6 de febrero del 87.

Mutis, J.C. (1793), El Arcano de la Quina. Revelado a beneficio de la humanidad. Discurso que contiene la parte médica.... Papel periódico de Santafé de Bogotá, ed. facsim. Bogotá, Banco de la Republica. 3: 285-604.

Mutis, J.C. (1828), El Arcano de la Quina, Madrid, Fundación de Ciencias de la Salud, Impresores de Cámara de S.M.

Mutis, J.C. (1957). Real proyecto del estanco de la quina y sus establecimientos. Reflexiones politicas que persuaden la suma importancia de erigir en Ramo de R. Hacienda la adinistración de la Quina... [Subtitulo del Informe del Dr. J. C. M. a S. M. el Rey Don Carlos IV, en 1786], Pp. 4464, En Pérez-Arbeláez, E. \& Fernández de Soto, F. (eds.) Flora de la Real Expedición Botánica del Nuevo Reino de Granada, vol. 44 .Ediciones Cultura Hispánica. Madrid.

Osorio, N. (1945). Sobre las quinas de los estados unidos de Colombia. Rev. Acad. Colomb. Ci. Exact. 6 (22): 244-273. 
Pérez-Arbeláez, E. (1998). El divino y amargo remedio. Pp. 167182. En: José Celestino Mutis. Su vida y su obra. Fondo FEN Colombia. Segunda edición. Bogotá. [Primera ed. 1967, Instituto Geogr. Agustín Codazzi].

Pérez-Arbeláez, E., Álvarez López, E., Uribe Uribe, L., Balguerías de Quesada, L., Sánchez Bella, A., Rivas Goday, S., Barras de Aragón, F., Concha, T. de la, Soriano, F. (1954). La Real Expedición Botánica del Nuevo Reino de Granada. Flora de la R. E. B. del N. R. de Granada, tomo I. [I-XIV, 144 p., 13 lám. col.] Ediciones Cultura Hispánica, Madrid.

Pérez-Arbeláez, E., Fernández de Soto Morales, F. (1957). Quinas de la Real Expedición Botánica del Nuevo Reino de Granada. Flora de la Real Expedición Botánica del Nuevo Reino de Granada Vol. 44. Instituto de Cultura Hispánica. Madrid.

RJB-CSIC. Biblioteca digital- e Icones Mutis, Real Jardín Botánico. Madrid (2019). https://bibdigital.rjb.csic.es/ records/item/15871-flora-de-la-real-expedicion-botanicadel-nuevo-reino-de-granada-t-44-quinas?offset=28 y http:// www.rjb.csic.es/icones/mutis/paginas/acercadelosdibujos.php

Ruiz, H. (1792). Quinología o tratado del árbol de la quina o cascarilla.... Madrid. Viuda e hijos de Marín.

Ruiz, H. \& J. Pavón. (1801). Suplemento a la Quinología en el qual se aumentan las especies de Quina... Madrid. Viuda e hijo de Marín.

San Pio (de), M.P. (2008). Mutis y la Real Expedición Botánica del Nuevo Reino de Granada. Real Jardín Botánico CSIC. Madrid, Editorial Lunwerb.

Standley, P.C. (1930). The Rubiaceae of Colombia. Field. Mus. Nat. Hist. Bot. Ser. 7 (1): 3-175.
Standley, P.C. (1931). The Rubiaceae of Ecuador. Field. Mus. Nat. Hist. Bot. Ser. 7 (2): 179-251.

Standley, P.C. (1936). Rubiaceae. In J.F. Macbride (ed.), Flora of Peru. Field. Mus. Nat. Hist. Bot. Ser. 3-261.

Steere, W.C. (1944). El descubrimiento y distribución de la Cinchona pitayensis en el Ecuador. Flora(Quito). 4: 1-9.

Thurland, N.J. \& al. (2018). Código Internacional de Nomenclatura para algas, hongos y plantas (Código de Shenzhen). Edición en español a cargo de W. Greuter y R.R. Rodriguez. Stiftung Herbarium Greuter, Berlín. Alemania. I-LII, $1-280 \mathrm{p}$,

Triana, J.J. (1872). Nouvelles études sur les quinquinas, d'après les matériaux présentés en 1867 à l' Exposition universelle de Paris et accompagnées de fac-similé des dessins de la quinologie de Mutis, suivis de remarques sur la culture de quinquinas, Paris.

Triana, J.J. (1937). Nuevos estudios sobre las quinas... Rev. Acad. Colomb. Ci. Exact. 1 (3): 257-275.

Triana, J.J. (1938a). Especies y variedades de las cinchonas de la “Quinología de Bogotá". Rev. Acad. Colomb. Ci. Exact. 2 (5): 67-103.

Triana, J.J. (1938b). Especies y variedades de las cinchonas de la "Quinología de Bogotá" (conclusión) Cultivo de las quinas. Rev. Acad. Colomb. Ci. Exact. 2 (7): 377-416.

Uribe Uribe, L. (1955). Pasifloráceas y Begoniáceas. Flora de la Real Expedición Botanica del Nuevo Reino de Granada tomo 27, Ediciones Cultura Hispánica. Madrid. I-IX, 1-135 p., Lam. 1-55, 1 map. col. 